\title{
Alvarado Score in Diagnosing Acute Appendicitis - Relevance
}

\author{
Dr. Krishna Kumar Mallick, Mr. Eric Yong Ngai Yin \\ MBBS(Gold Medalist), MS(General Surgery), FCGP(India), FSASMS(Delhi), MRSH(London). \\ Associate Professor, Surgery Unit, AIMST University, Malaysia. \\ MBBCh, BAO, MRCS, MS Hospital SultanahBahiyah, AlorSetar, Malaysia \\ Data compiled and references collected by:--- \\ 1. Angeline Tan Ee Ping 2. Chan Zi Yin 3. Chew Kok Jin 4. Lee Tze Hian \\ 5. Leong Kok Keong -- All MBBS from AIMST University.
}

\begin{abstract}
Acute appendicitis is a clinical diagnosis. But, because of various clinical presentations of this condition and numerous differential diagnosis, if appendectomy is done on merely clinical signs, some 15 to $30 \%$ of normal appendix would be removed.

The most globally accepted clinical and laboratory based diagnostic tool for acute appendicitis is 'Alvarado Score'. Accordingly a score of 7 or more is strongly predictive of acute appendicitis, whereas a score between 5 to 6 is suggestive of possible appendicitis and score below 5 is indicative of other causes of pain abdomen.(1)

In the present study, it has been tried to review all cases of acute appendicitis admitted in Hospital Sultanah Bahiyah, Alor Setar, Malaysia in 2012 and analyze them retrospectively to see the relevance of "Alvarado Score" in patients undergone appendectomy.

Abbreviation: HSB- - Hospital Sultanah Bahiyah, Alor Setar, Malaysia. AA- - Acute Appendicitis. AS- Alvarado Score. USG- - Ultrasonography.

Key Words: Acute appendicitis, Appendectomy, Alvarado Score, Diagnostic Tool.
\end{abstract}

\section{Introduction}

Acute appendicitis (AA) is the most common abdominal emergency in both developed and developing countries. (2) Accurate diagnosis of the condition is difficult, hence unaided clinical diagnosis of AA is unacceptable.(3) No imaging test is $100 \%$ correct in diagnosing AA.(4) Laparoscopic diagnosis is an invasive method and waiting for more sophisticated imaging results may lead both to cost-escalation and loss of precious time leading to increased morbidity and mortality to the patients. Abdominal ultrasonography is highly operatordependent test in diagnosing AA.(5) USG may give false negative results in perforated and gangrenous appendicitis.(6)

Clinical scoring system is a cheaper, faster and non-invasive diagnostic tool for AA. In 1986, Alvarado suggested a practical score consisting of 8 predictive factors to help in early diagnosis of AA. The Alvarado Score consists of symptoms, signs and laboratory investigations, the total having 10 points. According to Alvarado if the score is $=$ or $>$ than 7 , appendectomy should be done; if it is 5 to 6 , patient should be kept under observation, and if the score is $=$ or $<4$, other causes of pain abdomen should be looked for as there is very less chance of AA.(7) Many surgeons have found this score a sensible diagnostic tool for diagnosing AA and reducing negative appendectomy. $(8,9)$ Many researchers have found that AS has got a poor accuracy in Asian population in comparison to Western population. $(10,11)$ Some have seen that the false positive AS is very high in females in diagnosing AA. $(11,12,13,14,15)$ Some new scoring systems such as RIPASA Score and AIR(appendicitis inflammatory response) score have been developed and found to be more effective in comparison to ALVARADO Score. $(10,16,17)$

\section{AIMS AND OBJECTIVES}

1. To find out the effectiveness of AS in diagnosing AA by correlating it with the operative and histopathological findings.

2. To know the specificity and sensitivity of AS as a diagnostic tool of AA.

3. To find the ability of AS for using it as the only diagnostic tool in AA without any other investigative tools like abdomen ultrasonography and computed tomography.

4. To apply AS on the patients admitted for suspected AA.

5. To save the time and money by diagnosing AA with the help of AS, without using any extra-diagnostic imaging methods. 


\section{Materials and method}

All patients admitted in HSB in 2012 with suspicion of AA , based on significant history and positive findings on physical examination, were included in this study.

All these patients were individually rated, using AS (as per information obtained from the medical records and by cross checking with the admitting doctors). On this basis, the patients were divided into 3 groups:--

1. Patients with AS between 1 to 4 .

2. Patients with AS between 5 to 6 and

3. Patients with AS 7 or more than7 (7-10).

Alvarado Score Details

\begin{tabular}{|l|l|}
\hline Symptoms & Points \\
\hline Migratory right iliac fossa pain & 1 \\
\hline Anorexia & 1 \\
\hline Nausea and vomiting & 1 \\
\hline Signs & \\
\hline Right iliac fossa tenderness & 2 \\
\hline Rebound tenderness & 1 \\
\hline Fever & 1 \\
\hline Laboratory & 2 \\
\hline Leukocytosis & 1 \\
\hline Shift to left (segmented neutrophils) & 10 \\
\hline Total & 10 \\
\hline
\end{tabular}

In HSB the decision to go for appendectomy was solely based on the history taken by the concerned surgeon and on his clinical perception of the case. It was not necessarily based on AS.

Final diagnosis was made on the basis of histo-pathological examination of the excised appendix.

Relation between the AS and histo-pathological examination result has been seen by chi square test. The statistics is significant at $a=0.05$.

\section{Results}

In HSB 460 patients underwent appendectomy in 2012 on the basis of diagnosis of AA. The diagnosis was based on significant history, physical examination findings and laboratory investigations. 70 of these patients were excluded from the study as histo-pathology showed perforated appendicitis. So, the details of remaining study group are as follow:--

Total no. of study population(patients)---390

Total no. of male patients---178(45.64\%)

Total no. of female patients---212(54.36\%)

Positive histo-pathological diagnosis of AA---295(75.64\%)

Negative histo-pathology(No AA)---95(24.36\%)

Mean age of positive AA(295) cases---27.21 years.

Median age--- 23 years, the range being 4 to 83 years.

Total no. of male patients in positive AA (295) group---151(51.19\%)

Total no. of female patients in positive AA group ---144(48.81\%)

Total no. of male patients in negative AA (95) group---27(28.42\%)

Total no. of female patients in negative group $68 . .(71.58 \%)$

Taken male as the exposure, the risk ratio for AA is 1.25 . ( $\mathrm{P}=0.00017)$, as calculated by corrected chi square test.

Table 1

\begin{tabular}{|l|l|l|l|}
\hline & Final Diagnosis & & \\
\hline Gender & 0 & 2 & Total \\
\hline 1 & 144 & 68 & 212 \\
Row \% & $67.92 \%$ & $32.08 \%$ & $100 \%$ \\
Col \% & $48.81 \%$ & $71.58 \%$ & $54.36 \%$ \\
\hline 0 & 151 & 27 & 178 \\
Row\% & $84.83 \%$ & $15.17 \%$ & $100 \%$ \\
Col\% & $51.19 \%$ & $28.42 \%$ & $45.64 \%$ \\
\hline Total & 295 & 95 & 390 \\
Row\% & $75.64 \%$ & $24.36 \%$ & $100 \%$ \\
Col\% & $100 \%$ & $100 \%$ & $100 \%$ \\
& & & \\
& & & \\
& & &
\end{tabular}


Table 2

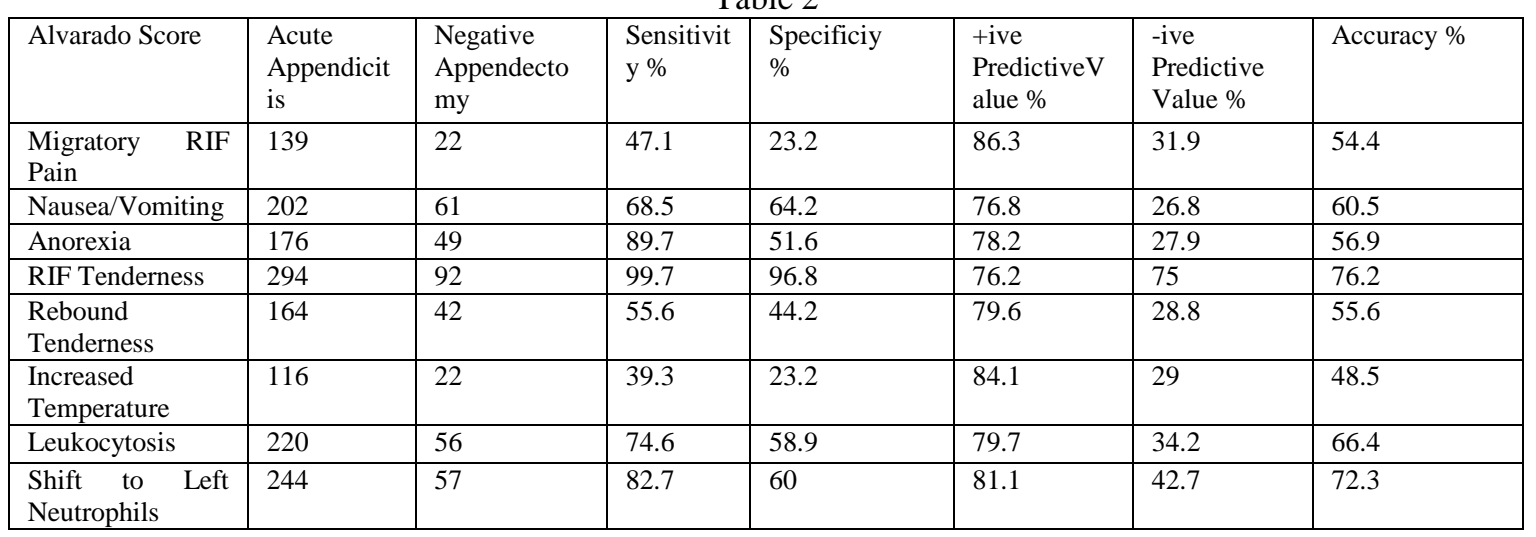

Table 3

\begin{tabular}{|c|c|c|c|c|c|}
\hline $\begin{array}{l}\text { Classification (On the } \\
\text { Basis of } \\
\text { AS) } \\
\text { A. }\end{array}$ & AA & $\begin{array}{l}\text { Negative } \\
\text { Appendectomy }\end{array}$ & $\begin{array}{l}\text { Sensitivity } \\
95 \% \text { CI }\end{array}$ & $\begin{array}{l}\text { Specificity } \\
95 \% \text { CI }\end{array}$ & $\begin{array}{l}\text { Diagnostic } \\
\text { Odds Ratio } \\
95 \% \text { CI }\end{array}$ \\
\hline Low Risk (0 to 4) & 59 & 45 & $20 \%(16$ to $25 \%)$ & $53 \%(43$ to $62 \%)$ & $\begin{array}{l}0.28 \text { (0.17 to } \\
0.46)\end{array}$ \\
\hline Medium Risk (5 to 6) & 107 & 29 & $36 \%(31$ to $42 \%)$ & $69 \%(60$ to $78 \%)$ & $\begin{array}{l}1.30(0.79 \text { to } \\
2.13)\end{array}$ \\
\hline High Risk(7 to 10$)$ & 129 & 21 & $44 \%(38$ to $49 \%)$ & $78 \%(69$ to $85 \%)$ & $\begin{array}{l}2.74 \text { (1.60 to } \\
4.68)\end{array}$ \\
\hline \multicolumn{6}{|l|}{ B. } \\
\hline Low Risk (0 to 4) & 59 & 45 & $20 \%(16$ to $25 \%)$ & $53 \%(43$ to $62 \%)$ & $\begin{array}{l}0.28 \text { (0.17 to } \\
0.46)\end{array}$ \\
\hline Medium Risk (5) & 47 & 13 & $16 \%(12$ to $21 \%)$ & $86 \%(78$ to $92 \%)$ & $\begin{array}{l}1.20(0.61 \text { to } \\
2.32)\end{array}$ \\
\hline High Risk (6 to 10) & 189 & 37 & $64 \%(58$ to $69 \%)$ & $61 \%(51$ to $70 \%)$ & $\begin{array}{l}2.80 \quad(1.74 \text { to } \\
4.5)\end{array}$ \\
\hline \multicolumn{6}{|r|}{ > } \\
\hline Low Risk (0 to 4$)$ & 59 & 45 & $20 \%(16$ to $25 \%)$ & $53 \%(43$ to $62 \%)$ & $\begin{array}{l}0.28 \text { (0.17 to } \\
0.46)\end{array}$ \\
\hline High Risk (5 to 10) & 236 & 50 & $80 \%(75$ to $84 \%)$ & $47 \%$ (38 to $57 \%)$ & $\begin{array}{lll}3.6 & (2.2 \text { to } \\
5.9) & & \end{array}$ \\
\hline
\end{tabular}

\section{Discussion}

The present study showed that 390 patients had been admitted to HSB in 2012 for appendectomy on the basis of significant history and physical findings. On histo-pathological results, 295(75.64\%) of them had been confirmed to have AA, while 95(24.36\%) of them had undergone negative appendectomy (wrong diagnosis).

The mean age was 27.21 years, which almost is comparable to the previous researchers.(18)

Of 95 patients with negative appendectomy, only 27(28.42\%) were male and rest 68 (71.58) were females. It, thus, shows that false positive results of Alvarado Scores are very high in female patients, as compared to male patients. So, in females additional investigations, like abdominal ultrasonography and CT scan, etc. should be mandatory. This finding is also comparable to the previous studies. $(12,13,14,15,16)$

Of all the 3 classifications of AS divided by using different cut-off value, classification C (low risk 0 to 4 , high risk 5 to 10) showed highest sensitivity $(80 \%, 90 \%$, Confidence Interval 75 to $84 \%)$ and specificity $(47 \%, 95 \%$, Confidence Interval 38 to 57\%) in the high risk group (compared to $44 \%$ and $78 \%$ of $1^{\text {st }}$ classification and 64 and $61 \%$ of $2^{\text {nd }}$ classification). After going into the depth it seems that we can improve the effectiveness of AS in diagnosing AA by implementing the new cut off value, i.e. low risk 0 to 4 and high risk 5 to 10 in local setting.

Of all the 8 components of AS, RIF tenderness is the most useful indicator (sensitivity $99.7 \%$, specificity $96.8 \%$, positive predictive value $76.2 \%$, negative predictive value $75 \%$ and accuracy $76.2 \%$ ). Other indicators of AS also produce high positive predictive value (76.8\% to $86.3 \%)$, but, unfortunately the negative predictive value is un-acceptably low (26.8\% to $42.7 \%)$. 


\section{Conclusion}

From this study, we concluded that in our local setting, efficacy (sensitivity, specificity and diagnostic odds ratio) of AS, using conventional cut off value of 7 for high risk group, in diagnosis of AA is disappointing. However, by using the new cut off value of 5 for high risk group, the efficacy of AS in diagnosing AA can be greatly enhanced and then totally acceptable as a diagnostic tool. But, in cases of acute abdomen in women an additional test like USG or CT Scan of abdomen should be done to exclude other causes, because of high false positive result.

\section{References}

[1]. Norman S. Williams et al Bailey\& Love's Short Practice of Surgery. Hodder Arnold, 25 ${ }^{\text {th }}$ ed.2008, Pg. 1206-18

[2]. Okobia MN et al. Acute Appendicitis: Review of the rate of negative appendectomy in Benin City. Nig. J Surg 1999; 6: 1-5.

[3]. Hoffman J et al. Diagnostic Aids in Acute Appendicitis. Br J Surg 1989; 76(8): 774-9.

[4]. Field S. et al. Acute Abdomen. In Sutton D Textbook of Radiology and Imaging. Churchill Livingston, $7^{\text {th }}$ ed. 2003 . Pg 685.

[5]. John $\mathrm{H}$ et al. Comparison of Clinical Judgment \& Diagnostic Ultrasonography in the Diagnosis of Acute Appendicitis: experience with a score-aids diagnosis. Eur J Surg 1997; 163(6): 433-43.

[6]. Charles D Douglas et al. Controlled Trial of Ultrasonography in Diagnosis of Acute Appendicitis incorporating the Alvarado Score. BMJ 2000; 321(7266): 919.

[7]. Alfredo Alvarado MD. A Practical Score for the early diagnosis of Acute Appendicitis. Ann Emerg Med 1986; 15(5): 557-564.

[8]. TD Owen et al. Evaluation of Alvarado Score in Acute Appendicitis. J Soc Med 1992; 85(2): 87-88.

[9]. Kanumba ES et al. Modified Alvarado Scoring System as a Diagnostic Tool for Acute Appendicitis at Bugando Medical Centre, Mwanza, Tanzania. BMJ Surg 2011; 11:4

[10]. Chong CF, et al. Evaluation of the RIPASA Score: a new scoring system for the acute appendicitis. Brunei. Int. Med J 2010; 6(1):17-26.

[11]. Shrivastava UK, et al. Evaluation of the Alvarado Score in the Diagnosis of Acute Appendicitis. Trop Gastroenterol 2004; 25(4): 184-6

[12]. M.Kalan et al. Evaluation of the Modified Alvarado Score in the Diagnosis of Acute Appendicitis: a prospective study. Ann R CollSurgEngl 1994;76(6): 418-9

[13]. Malik AA et al. Continuing Diagnostic Challenge of Acute Appendicitis. Aust NZ J Surg 1998; 68(7): 504-5

[14]. Bhattachajee PK et al. Prospective Evaluation of Modified Alvarado Score for diagnosis of Acute Appendicitis. J Ind Med Assoc 2002;100(5):310-14

[15]. Menon ZA et al. Acute Appendicitis : Diagnostic Accuracy of Alvarado Scoring System. Asian J Surg 2013 (EPub ahead of print) Accessed@www.ncbi.nlm.nih.gov/pubmed/25726829

[16]. Chong CF et al. Comparison of RIPASA \& Alvarado Scores for the diagnosis of Acute Appendicitis. Sing Med J 2011;52(5):340-5

[17]. I Alnjadat et al. Alvarado versus RIPASA Score in Diagnosing Acute Appendicitis. Rawal Med J 2013;38(2):147-151

[18]. P Jeerapa, T Taweesak. The Modified Alvarado Score versus the Alvarado Score for the diagnosis of acute Appendicitis. The Thai $\mathrm{J}$ Surg 2005;26:69-72 